\title{
New Method for Basic Detection and Characterization of Flaws in Composite Slabs through Finite Difference Thermal Contrast (FDTC)
}

by Andrés Restrepo G.* and Humberto Loaiza C.*

*Facultad de Ingeniería, Universidad Santiago de Cali. PhD candidate, Universidad del Valle, Cali, Colombia, adareg378@gmail.com

**Escuela de Ingeniería Eléctrica y Electrónica, Universidad del Valle, Cali, Colombia, hloaiza@univalle.edu.co

\section{Abstract}

In this paper, a technique based on a new model from finite differences discretization of Fourier heat propagation in $3 \mathrm{D}$, is presented in order to be applied on a sequence of infrared images to enhance contrast and automatically detect and characterize flaws in composite slabs. The performance of this technique is evaluated using artificial thermal sequences from a simulated CFRP slab generated by ThermoCalc6L software. Results show that this technique offers a better contrast between defect and background than other common techniques like modified differentiated absolute contrast, and it runs faster than the classic 3D thermal filtering method.

\section{Introduction (Arial, 9pt, bold)}

Pulse thermography (PT) is a non-destructive evaluation (NDE) technique that has become one of the most important procedures in the aeronautical and automotive industry for analysing composite materials like carbon fibre reinforced plastic (CFRP), because it is faster and easier to develop than other thermography methods. In an experiment of PT, a flash is used to heat the material under inspection, and the information consists of a sequence of IR images, from which the cooling profiles at every pixel can be extracted $[1,2,3]$. Nevertheless, flashing the material makes the heating pulse be spatially non-uniform [4], and so, the background thermal information is not compensated in a suitable way if a classic contrast enhancement technique is used, mainly because of the lateral diffusion of heat [5]. Methods like differentiated absolute contrast (DAC) or the more complete modified-DAC [6] have, as a principal drawback, the use of the 1-dimensional model of heat transfer to deal with a 3-dimensional problem.

In general, the multidimensional processing has not been extensively applied in active IR thermography (IT), and since few years it begins to be used in noise reduction [7], thermal data compression [8], simulation of temperature distribution and contrast enhancement [5], and solution of inverse problems [9]; however, in simulation and inversion tasks, working with 3D propagation model leads to very heavy algorithms from a computationally point of view. In that scenario, we propose a method which is based on this 3D model, but unlike others, does not perform temperature estimation in three spatial axes throughout the material in every sample time: for each pixel, it estimates the value of temperature in the next time, only on the frontal surface of the specimen, from actual temperature data of the same pixel and its neighbours. This feature makes this technique to be faster and suitable to implement as a convolution filter for images. Moreover, it does not need to select a sound area a priori, like in former contrast enhancement techniques, or a reference image, like in techniques derived from DAC [10].

\section{3D heat propagation model and finite differences discretization}

The complete 3D model of heat propagation through a piece of material is expressed by Eq. (1) $[11,12]$ :

$$
\frac{\partial}{\partial x}\left(\kappa_{x} \frac{\partial T(x, y, z, t)}{\partial x}\right)+\frac{\partial}{\partial y}\left(\kappa_{y} \frac{\partial T(x, y, z, t)}{\partial y}\right)+\frac{\partial}{\partial z}\left(\kappa_{z} \frac{\partial T(x, y, z, t)}{\partial z}\right)+w(x, y, z, t)=C \rho \frac{\partial T(x, y, z, t)}{\partial t}
$$

where:

- $\quad x, y, z, t:$ spatial coordinates (with senses of figure 1) and time;

- $\quad T=T(x, y, z, t)$ : excess of temperature respect to room temperature, according to spatial coordinates and time;

- $\quad C, \rho$ : specific heat and density;

- $\kappa_{q}$ : thermal conductivity in the $q$ direction;

- $\quad w(x, y, z)$ : heat generation rate by volume unity $[\mathrm{W} / \mathrm{m} 3]$ inside the propagation medium.

- $Q(x, y, z, t)$ : power density of absorbed thermal flux. 


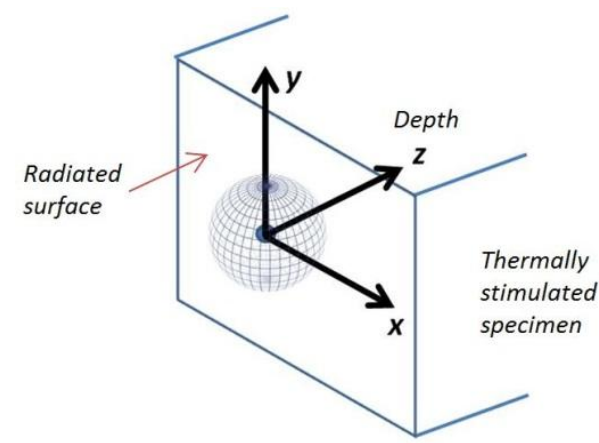

Fig. 1. Conventional senses of each spatial coordinate

If we consider that the specimen is an anisotropic and homogenous medium, thermal excitation is very similar to an impulse function, no heat is generating inside the slab, and there is no loses by convection through its surfaces, Eq. (1) can be discretized by finite differences in an explicit way, as shown by Eq. (2):

$$
\frac{1}{\alpha} \frac{T_{i j k}^{m+1}-T_{i j k}^{m}}{\Delta t}=\left(\frac{T_{(i-1) j k}^{m}-2 T_{i j k}^{m}+T_{(i+1) j k}^{m}}{\Delta x^{2}}\right)+\left(\frac{T_{i(j-1) k}^{m}-2 T_{i j k}^{m}+T_{i(j+1) k}^{m}}{\Delta y^{2}}\right)+\left(\frac{T_{i j(k-1)}^{m}-2 T_{i j k}^{m}+T_{i j(k+1)}^{m}}{\Delta z^{2}}\right)
$$

where:

- $t_{m}=m . \Delta t$, con $m=0,1,2, \ldots, M-1$, being $M$ the total number of thermograms acquired, and $\Delta t$, acquisition period of images;

- $\quad x_{i}=i . \Delta x$, con $i=0,1,2, \ldots, N_{x}-1$, being $N_{x}$ the total number of rows in each image; and $\Delta x=\frac{L_{x}}{N_{x}}$, being $L_{x}$ the vertical length of image field;

- $y_{i}=j . \Delta y$, con $j=0,1,2, \ldots, N_{y}-1$, being $N_{y}$ the total number of columns in each image; and $\Delta y=\frac{L_{y}}{N_{y}}$, being $L_{y}$ the horizontal length of image field;

- $\quad z_{k}=k . \Delta z$, con $k=0,1,2, \ldots, N_{k}-1$, being $N_{k}$ the total number of steps dividing the thickness of slab; and $\Delta z=\frac{L_{z}}{N_{z}}$, being $L_{z}$ that thickness. Value of $N_{k}$ is unknown in a real Pulse Thermography experiment;

- $\quad T_{i j k}^{m}$, temperature on pixel $i, j, k$ for thermogram $m$;

- $\alpha=\frac{K}{C \rho}$ being the thermal diffusivity of the specimen.

Considering a pixel as a squared element, so $\Delta x=\Delta y=\Delta p$, and defining a dimensionless parameter: $\beta=\alpha \frac{\Delta t}{\Delta p^{2}}$, we have:

$$
T_{i j k}^{m+1}-T_{i j k}^{m}=\beta\left[\left(T_{(i-1) j k}^{m}-2 T_{i j k}^{m}+T_{(i+1) j k}^{m}\right)+\left(T_{i(j-1) k}^{m}-2 T_{i j k}^{m}+T_{i(j+1) k}^{m}\right)\right]+\left(\frac{T_{i j(k-1)}^{m}-2 T_{i j k}^{m}+T_{i j(k+1)}^{m}}{\Delta z^{2}}\right)
$$

Rearranging terms:

$$
T_{i j k}^{m+1}=(1-4 \beta) T_{i j k}^{m}+\beta\left(T_{(i-1) j k}^{m}+T_{(i+1) j k}^{m}+T_{i(j-1) k}^{m}+T_{i(j+1) k}^{m}\right)+\beta \frac{\Delta p^{2}}{\Delta z^{2}}\left(T_{i j(k-1)}^{m}-2 T_{i j k}^{m}+T_{i j(k+1)}^{m}\right)
$$

Finally, grouping terms with $T_{i j k}^{m+1}$ and defining: $\Phi_{k}^{m}=T_{(i-1) j k}^{m}+T_{(i+1) j k}^{m}+T_{i(j-1) k}^{m}+T_{i(j+1) k}^{m}$, as the sum of temperatures of 4-neighbors of pixel $i, j, k$ at instant $m$ :

$$
T_{i j k}^{m+1}=\left[1-2 \beta\left(2+\frac{\Delta p^{2}}{\Delta z^{2}}\right)\right] T_{i j k}^{m}+\beta\left[\Phi_{k}^{m}+\frac{\Delta p^{2}}{\Delta z^{2}}\left(T_{i j(k-1)}^{m}+T_{i j(k+1)}^{m}\right)\right]
$$

Knowing $\beta$, and choosing $\Delta z$ so that $\alpha \frac{\Delta t}{\left(\Delta z^{2}\right)}<\frac{1}{4}$ (by reasons of model stability [13]), the unknown terms $T_{i j(k-1)}^{m}$ and $T_{i j(k+1)}^{m}$ represent the only problem to estimate the next value of temperature on pixel $i, j, k$. So, two approximations to these terms are proposed using two exponential models being solutions of Fourier differential equation applying Green functions $[14,15,16]$, and assuming that heating lateral dispersion is negligible for a thermal propagation of $\Delta z$. 


\subsection{First approximation}

The models proposed by Tadeu and Simões [15] can be decomposed in two classes of terms: one class represents the influence of heat source; and the second one, the influence of border temperature and flux conditions (if convection effects are neglected). For a slab case, and conditions concerning an active IT experiment, it can be established that heat fluxes are null on both sides of the layer, for which the temperature at point $x, y, z$ inside the slab (of width $h$ ), due to a heat point source placed at $x_{0}, y_{0}, Z_{0}$, is determined by:

$$
T(x, y, z, t)=\frac{W e^{-r_{0} / 4 \alpha \tau}}{\rho C(4 \pi \alpha \tau)^{3 / 2}}+\sum_{n=1}^{N S} \frac{e^{-r_{1} / 4 \alpha \tau}+e^{-r_{2} / 4 \alpha \tau}+e^{-r_{3} / 4 \alpha \tau}+e^{-r_{4} / 4 \alpha \tau}}{\rho C(4 \pi \alpha \tau)^{3 / 2}}
$$

where:

$$
\begin{aligned}
& r_{0}=\left(x-x_{0}\right)^{2}+\left(y-y_{0}\right)^{2}+\left(z-z_{0}\right)^{2} ; r_{1}=\left(x-x_{0}\right)^{2}+\left(y-y_{0}\right)^{2}+\left(z+z_{0}+2 h(n-1)\right)^{2} ; \\
& r_{2}=\left(x-x_{0}\right)^{2}+\left(y-y_{0}\right)^{2}+\left(z+z_{0}-2 h n\right)^{2} ; r_{3}=\left(x-x_{0}\right)^{2}+\left(y-y_{0}\right)^{2}+\left(z-z_{0}+2 h n\right)^{2} ; \\
& r_{4}=\left(x-x_{0}\right)^{2}+\left(y-y_{0}\right)^{2}+\left(z-z_{0}-2 h n\right)^{2}
\end{aligned}
$$

Because the idea is to obtain an approximation that takes into account only the temperature distribution throughout $z$ axis for a $\Delta z$ displacement, Eq. (4) can be rewritten as:

$$
T\left(z_{k}, t_{m}\right)=\frac{W}{\rho C(4 \pi \alpha \tau)^{1 / 2}}\left[e^{-r_{0}^{\prime} / 4 \alpha \tau_{m}}+\sum_{n=1}^{N S}\left(e^{-r_{1}^{\prime} / 4 \alpha \tau_{m}}+e^{-\dot{r}_{2}^{\prime} / 4 \alpha \tau_{m}}+e^{-\dot{r}_{3} / 4 \alpha \tau_{m}}+e^{-\dot{r}_{4}^{\prime} / 4 \alpha \tau_{m}}\right)\right]
$$

where:

$$
\begin{aligned}
& r_{0}^{\prime}=\left(z_{k}-z_{0}\right)^{2} ; r_{1}^{\prime}=\left(z_{k}+z_{0}+2 h(n-1)\right)^{2} ; r_{2}^{\prime}=\left(z_{k}+z_{0}-2 h n\right)^{2} ; \\
& r_{3}^{\prime}=\left(z_{k}-z_{0}+2 h n\right)^{2} ; \dot{r}_{4}^{\prime}=\left(z_{k}-z_{0}-2 h n\right)^{2}
\end{aligned}
$$

In Eqs. (4) and (5), NS is an arbitrary number of thermal wave reflections to be considered (the smaller diffusivity, the higher $N S$ value). Now, assuming that heat pulse is applied at initial time $\left(t_{0}=0\right)$ and over the frontal surface $\left(z_{0}=0\right)$ of the slab under inspection, and defining: $\sigma_{m}=4 \alpha t_{m}=4 \beta m \Delta p^{2}$, we can write:

being:

$$
T_{i j k}^{m}=\frac{W}{\rho C\left(4 \pi \alpha t_{m}\right)^{1 / 2}}\left[A_{k}^{(N S)}{ }_{k}^{m}\right]
$$

$$
A_{k}^{(N S)_{k}^{m}}=e^{-z_{k}^{2} / \sigma_{m}}+\sum_{n=1}^{N S}\left(e^{-\left(z_{k}+2 h(n-1)\right)^{2} / \sigma_{m}}+2 e^{-\left(z_{k}-2 h n\right)^{2} / \sigma_{m}}+e^{-\left(z_{k}+2 h n\right)^{2} / \sigma_{m}}\right)
$$

that:

To eliminate the constant due to power density of heating $W$ in the approximation, it can be defined in general

$$
T_{i j(k \pm 1)}^{m}=\frac{T_{i j k}^{m}}{A^{(N S)_{k}^{m}}}\left[A^{(N S)_{k \pm 1}^{m}}\right]
$$

So, replacing Eq. (8) in Eq. (3), we obtain:

$$
\tilde{T}_{i j k}^{m+1}=\left\{1-\beta\left[4+\frac{\Delta p^{2}}{\Delta z^{2}}\left(2-\frac{\left(A^{(N S)}{ }_{k+1}^{m}+A^{(N S)}{ }_{k-1}^{m}\right)}{A^{(N S)_{k}^{m}}}\right)\right]\right\} T_{i j k}^{m}+\beta \Phi_{k}^{m}
$$

Since the information acquired from thermal sensor corresponds to surface temperature, where $z=0(k=0)$, then we have finally the $3 D$ differentiated thermal estimation proposed:

$$
\tilde{T}_{i j 0}^{m+1}=\left\{1-\beta\left[4+\frac{\Delta p^{2}}{\Delta z^{2}}\left(2-\frac{\left(A^{(N S)_{(1)}^{m}+A_{(N S)}^{(m)}}{ }_{(-1)}\right)}{A^{(N S)_{0}^{m}}}\right)\right]\right\} T_{i j 0}^{m}+\beta \Phi_{0}^{m}
$$

with:

$$
\Phi_{0}^{m}=T_{(i-1) j 0}^{m}+T_{(i+1) j 0}^{m}+T_{i(j-1) 0}^{m}+T_{i(j+1) 0}^{m}
$$

\subsection{Second approximation}

The second approximation to $T_{i j(k-1)}^{m}$ and $T_{i j(k+1)}^{m}$ consists in using the Tadeu and Simões model for a semiinfinite medium, with null normal flux at the surface $(z=0)$ : 
where:

$$
T(x, y, z, t)=W \cdot \frac{e^{-r_{0} / 4 \alpha \tau}+e^{-r_{1} / 4 \alpha \tau}}{\rho C(4 \pi \alpha \tau)^{3 / 2}}
$$

$$
r_{0}=\left(x-x_{0}\right)^{2}+\left(y-y_{0}\right)^{2}+\left(z-z_{0}\right)^{2} ; r_{1}=\left(x-x_{0}\right)^{2}+\left(y-y_{0}\right)^{2}+\left(z+z_{0}\right)^{2} .
$$

Taking again the temperature distribution only throughout $z$ axis, and the source applied at $z_{0}=0$ and $t_{0}=0$, results:

$$
T\left(z_{k}, t_{m}\right)=W \cdot \frac{2 e^{-z_{k}^{2} / 4 \alpha t_{m}}}{\rho C(4 \pi \alpha)^{1 / 2} t_{m}^{1 / 2}}
$$

In a similar way than first approximation, the information about power density $\mathrm{W}$ can be discarded, knowing from Eq. (13) that:

$$
\frac{2 \mathrm{~W}}{\rho C(4 \pi \alpha)^{1 / 2} t_{m}^{1 / 2}}=T\left(z_{k}, t_{m}\right) e^{z_{k}{ }^{2} / 4 \alpha t_{m}}
$$

So, it can be written:

$$
T_{i j(k \pm 1)}^{m}=T_{i j k}^{m} e^{(k \Delta z)^{2} / 4 \beta m \Delta p^{2}} e^{-((k \pm 1) \Delta z)^{2} / 4 \beta m \Delta p^{2}}
$$

Replacing Eq. (14) in Eq. (3), results:

$$
\tilde{T}_{i j k}^{m+1}=\left\{1-2 \beta\left[2+\frac{\Delta p^{2}}{\Delta z^{2}}\left(1-e^{(k \Delta z)^{2} / 4 \beta m \Delta p^{2}}\left(e^{-((k+1) \Delta z)^{2} / 4 \beta m \Delta p^{2}}+e^{-((k-1) \Delta z)^{2} / 4 \beta m \Delta p^{2}}\right)\right)\right]\right\} T_{i j k}^{m}+\beta \Phi_{k}^{m}
$$

Restricting Eq. (15) for temperature data at surface $(k=0)$, we obtain finally:

$$
\tilde{T}_{i j 0}^{m+1}=\left\{1-2 \beta\left[2+\frac{\Delta p^{2}}{\Delta z^{2}}\left(1-e^{-\Delta z^{2} / 4 \beta m \Delta p^{2}}\right)\right]\right\} T_{i j 0}^{m}+\beta \Phi_{0}^{m}
$$

\section{Finite difference thermal contrast}

The technique named finite difference thermal contrast (FDTC) basically consists in arranging the terms of Eq. (10) (or Eq. (16)) in a convolution mask or kernel, as shown in figure 2, in such a way that can be applied to the thermal sequence like a spatial filtering, to obtain a contrasted sequence in which defects show up and thermal background is compensated. The underlying idea is that the relative error in the temperature estimation, at next time for each pixel, will be greater in those zones where flaws arise. Because of that, the output $F_{i j 0}^{m}$ of this filter is defined by Eq. (17).

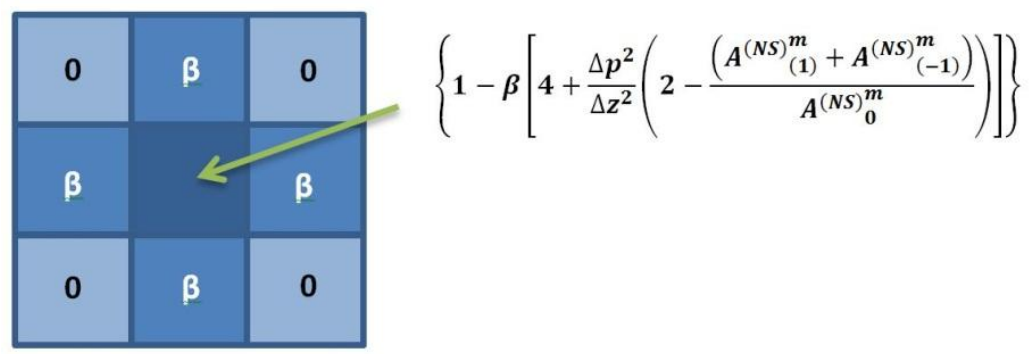

Fig. 2. Filter mask for FDTC with $1^{\text {st }}$ approximation. In a similar way, it can be used for FDTC with $2^{\text {nd }}$ approximation

$$
F_{i j 0}^{m}=\frac{T_{i j 0}^{m+1}-\widetilde{T}_{i j 0}^{m+1}}{T_{i j 0}^{m+1}}
$$

Clearly, this is a dynamic filter in the sense that the mask change for one pixel to another; however, the FDTC algorithm does not pretend to rebuild the temperature distribution throughout the thickness of the slab, but estimates the temperature values only at the surface, executing the filtering for all of thermal images. This feature does the FDTC technique to run faster than any processing method based on 3D thermal reconstruction in space. 
A CFRP slab with 9 internal squared flaws of air, shown in figure 3, was synthetized by using ThermoCalc6L software with parameters listed in tables 1 and 2. From the simulated images sequence from that layer, a set of thermal profiles were extracted, taking the centroids of the defects No. 1, 2, 3, 4 and 7, and a sound point after applying the FDTC filtering; these profiles are shown in figures 4 and 5.

Table 1. Simulation parameters for PT on artificial CFRP slab

\begin{tabular}{|c|c|c|c|c|c|c|c|c|c|c|c|c|}
\hline $\begin{array}{c}\text { Length } \\
{[\mathrm{m}]}\end{array}$ & $\begin{array}{l}\text { Width } \\
{[\mathrm{m}]}\end{array}$ & $\begin{array}{c}\text { Thick } \\
{[\mathrm{m}]}\end{array}$ & $\Delta \mathbf{x}[\mathrm{m}]$ & $\Delta y[\mathrm{~m}]$ & $\Delta z[\mathrm{~m}]$ & $\begin{array}{c}\mathrm{K} \\
{[\mathrm{W} / \mathrm{m} . \mathrm{K}]}\end{array}$ & $\underset{[W . s / K g . K]}{C}$ & $\left.\begin{array}{c}\rho \\
{[\mathrm{Kg} / \mathrm{m} 3]}\end{array}\right]$ & $\Delta t[s]$ & $\begin{array}{l}\text { Acquisition } \\
\text { window [s] }\end{array}$ & $\begin{array}{c}\text { Pulse } \\
\text { density } \\
{[W / m 2]}\end{array}$ & $\begin{array}{c}\text { Pulse } \\
\text { width } \\
\text { [s] }\end{array}$ \\
\hline 0.2 & 0.2 & 0.002 & 0.001 & 0.001 & 0.0001 & 0.7 & 1200 & 1600 & 0.0063 & 7 & 100000 & 0.01 \\
\hline
\end{tabular}

Table 2. Features of simulated internal defects

\begin{tabular}{|c|c|c|c|c|c|c|}
\hline Defect No. & K [W/m.K] & C [W.s/Kg.K] & $\rho$ [Kg/m3] & Side [m] & Depth [m] & Thick [m] \\
\hline $1-2-3$ & 0.07 & 928 & 1.3 & $\begin{array}{c}0.015-0.010 \\
-0.005\end{array}$ & 0.0002 & 0.0001 \\
\hline $4-5-6$ & 0.07 & 928 & 1.3 & $\begin{array}{c}0.015-0.010 \\
-0.005\end{array}$ & 0.0006 & 0.0001 \\
\hline $7-8-9$ & 0.07 & 928 & 1.3 & $\begin{array}{c}0.015-0.010 \\
-0.005\end{array}$ & 0.0010 & 0.0001 \\
\hline
\end{tabular}

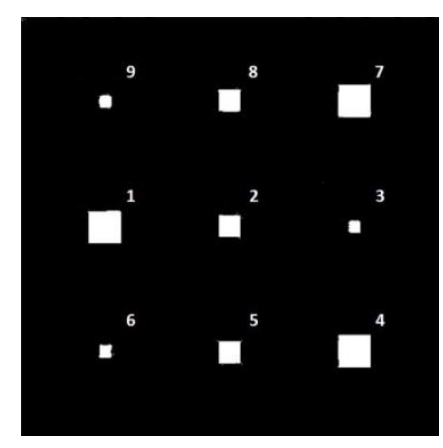

Fig. 3. Synthetic CFRP slab with 9 internal flaws

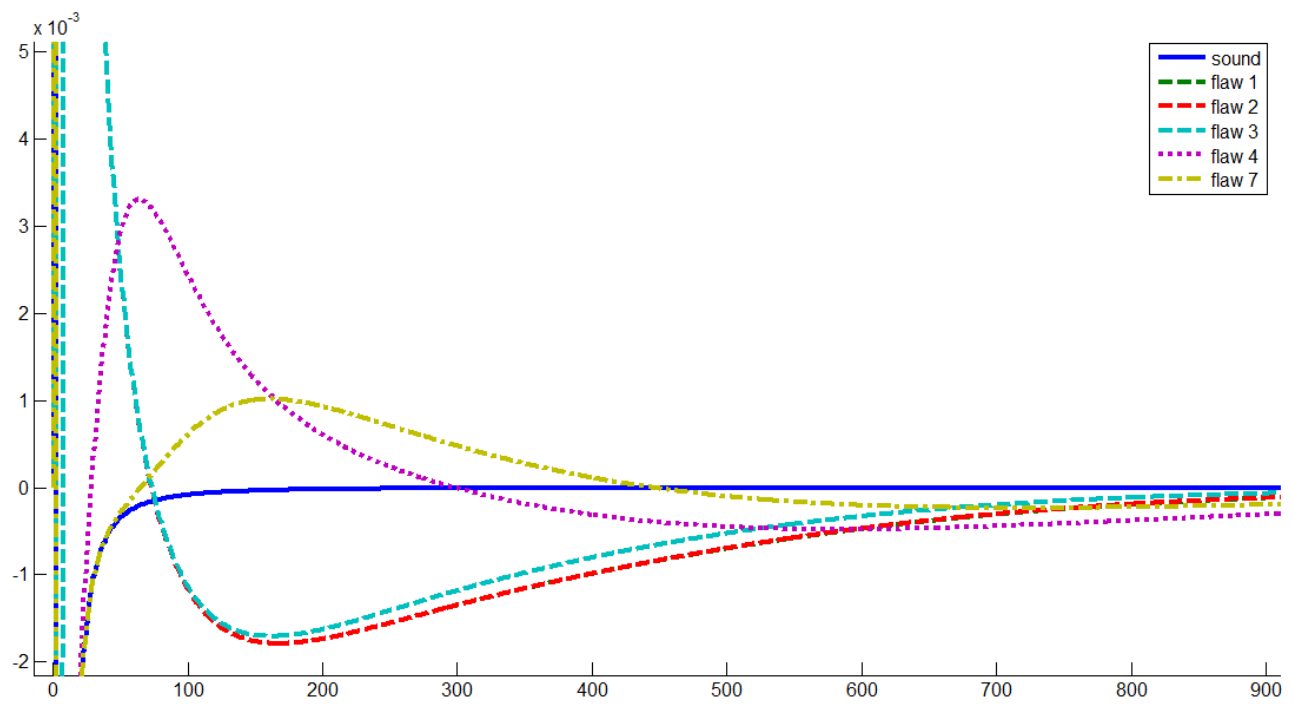

Fig. 4. Differential profiles for defects 1,2, 3, 4 and 7, and a sound point, during the first 5.7s after FDTC filtering with $1^{\text {st }}$ approximation (zoom view). 


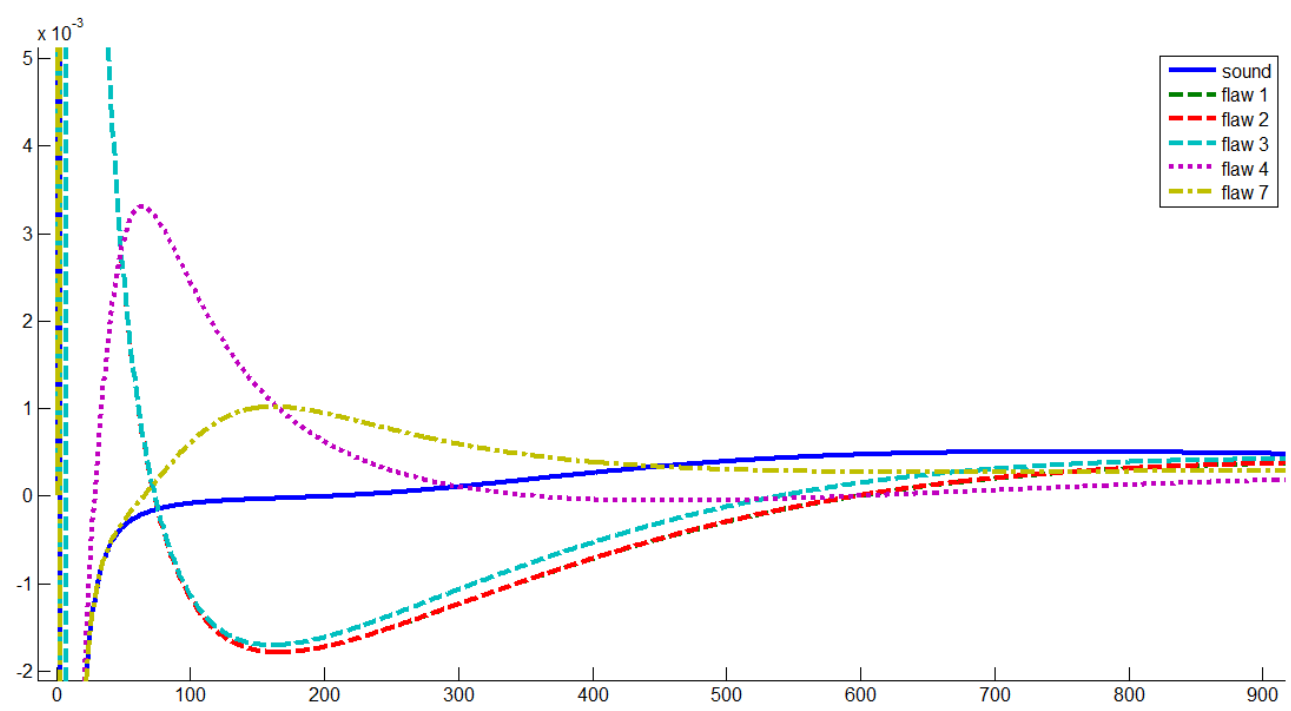

Fig. 5. Differential profiles for defects 1, 2, 3, 4 and 7, and a sound point, during the first 5.7s after FDTC filtering with $2^{\text {nd }}$ approximation (zoom view).

We can observe in figure 4 that the sound profile has the lowest energy, while the defect profiles deviate, first above and later below the sound profile. This behaviour is according to an initial slower cooling phase and a later faster cooling phase of the defect regions respect to sound regions; this is true for flaw conductivity smaller than layer conductivity. Also, profiles for flaws 1, 2 and 3 (at the same depth) tend to merge in this graph, while the more deep flaws have profiles with farther peaks in time; this fact evidence a correlation between depth of defects and peak values of its thermal profiles. Furthermore, it is noticeable a spurious transient oscillation at the beginning of profiles, due probably to discrepancies of initial conditions, and a heat pulse that is not an impulse $\delta(t)$. Meanwhile, it is clear in figure 5 that, when the approximation for a semi-infinite medium is used, all profiles tend to rise for longer times due to the back surface effect of the slab; but this effect is useful to estimate the depth of flaws, as it will be seen in the next chapter.

Finally, for detection purpose, it is better to work with the accumulated profiles (Eq. (18), where $P_{D}$ and $P_{A}$ are respectively the accumulated and differential profiles), as seen in figure 6 . Here, the amplitude decay of defect profiles when the flaw depth increase is less than the amplitude decay obtained with other techniques, fact that becomes a factor for better global contrast.

$$
P_{A}(i, j, t)=\sum_{\tau=1}^{t} P_{D}(i, j, \tau)
$$

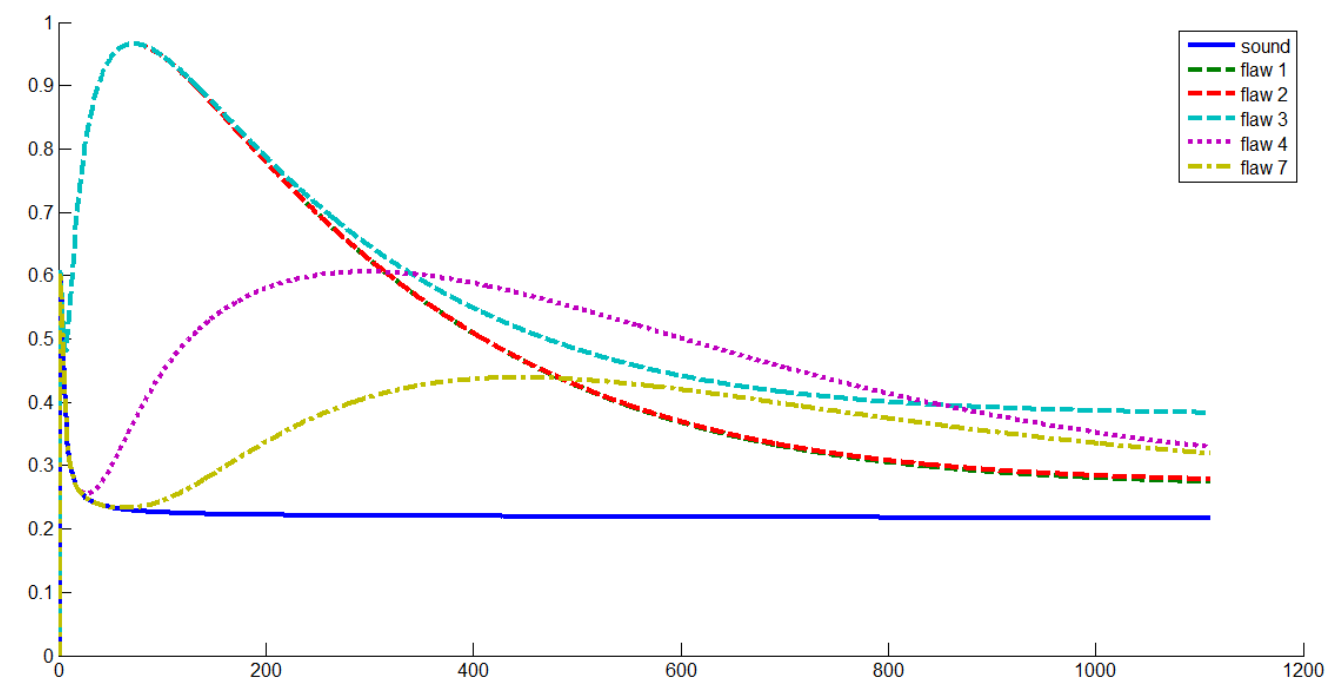

Fig. 6. Accumulated profiles for defects 1, 2, 3, 4 and 7, and a sound point, during 7s after FDTC filtering with $1^{\text {st }}$ approximation. 


\section{Characterization}

The relation between the squared depth of a flaw $z_{d}$ and diffusivity $\alpha$ of the specimen is known to be equivalent to the transit time or diffusion time $t_{t r}$, at which the defect region begins to contrast from the image background [11]. In [17] the Laplace transform is used to model a biological system consisting of three layers (with depths $z_{a}, z_{b}, z_{c}$ and diffusivities $\alpha_{0}, \alpha_{1}, \alpha_{2}$ ), which together behave as an only layer in a transient state, with an effective diffusivity $\alpha_{\text {eff }}$ defined by Eq. (19):

$$
\alpha_{e f f}=1 /\left(\frac{z_{a}}{L \sqrt{\alpha_{0}}}+\frac{z_{b}}{L \sqrt{\alpha_{1}}}+\frac{z_{c}}{L \sqrt{\alpha_{2}}}\right)^{2} ; \quad L=z_{a}+z_{b}+z_{c}
$$

Similarly, attending the scheme in figure 7, about a piece of material (diffusivity $\alpha$ ) with an internal defect (diffusivity $\alpha_{d}$ ), we realize that Eq. (19) can be applied in this situation, and write:

$$
\frac{z_{L}}{\sqrt{\alpha_{e f f}}}=\frac{z_{1}}{\sqrt{\alpha}}+\frac{z_{2}}{\sqrt{\alpha_{d}}}+\frac{z_{3}}{\sqrt{\alpha}} \quad ; \quad z_{L}=z_{1}+z_{2}+z_{3}
$$

But Eq. (20) is equivalent to:

$$
\sqrt{t_{\text {treff }}}=\sqrt{t_{\text {tr } 1}}+\sqrt{t_{\text {tr } 2}}+\sqrt{t_{\text {tr } 3}}
$$

So:

$$
\frac{\sqrt{t_{\text {tr } 1}}}{\sqrt{t_{\text {treff }}}}=\frac{z_{1}}{\sqrt{\alpha}} / \frac{z_{L}}{\sqrt{\alpha_{\text {eff }}}}
$$

If $z_{2}<<z_{1}+z_{3}$ or $\alpha_{2}>>\alpha$, then $\alpha_{\text {eff }} \approx \alpha$, and:

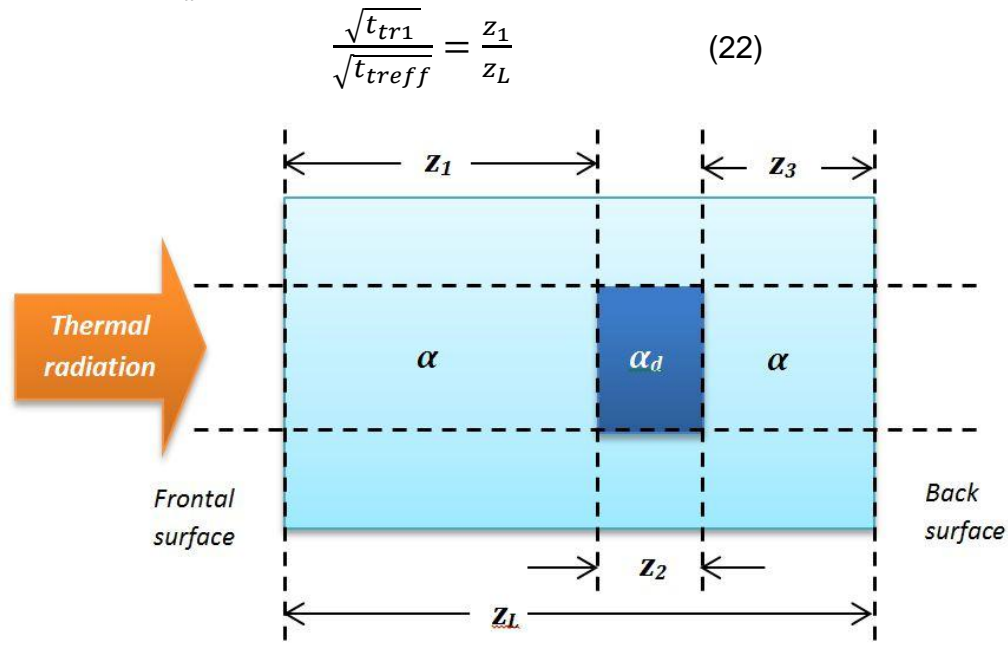

Fig. 7. Scheme of diffusion problem through an internal defect.

Given the correlation between depth of a flaw and the peak of its thermal profile, and the correlation of the end of slab (back surface) and the presence of a peak in a sound profile, we can rewrite Eq. (22) to replace diffusion times $t_{t r 1}$ and $t_{\text {treff }}$ by the times $t_{d}^{\max }$ and $t_{L}{ }^{\max }$ when respective profiles reach their maximum values, to obtain:

$$
z_{d}=z_{L} \frac{\sqrt{t_{d}^{\max }}}{\sqrt{t_{L}^{\max }}}
$$

\section{Results}

In figures $8 \mathrm{a}, \mathrm{b}, \mathrm{c}$ and $\mathrm{d}$, is shown the spatial profile along the segment joining the centroids of defects No. 1, 2 and 3 , for the thermogram at $t=5 \mathrm{~s}$, extracted from the original simulated sequence, the DAC-m sequence, the FDTC $\left(1^{\text {st }}\right.$ approx.) sequence, and the truncated FDTC ( $1^{\text {st }}$ approx.) sequence. In figure $8 \mathrm{c}$ the borders of flaws are more abrupt than in figure $8 \mathrm{~b}$, but they may experiment a strong oscillation around the background level; for that, in the last thermogram the background level is set to zero, and so, the resulting negative values level are truncated. 

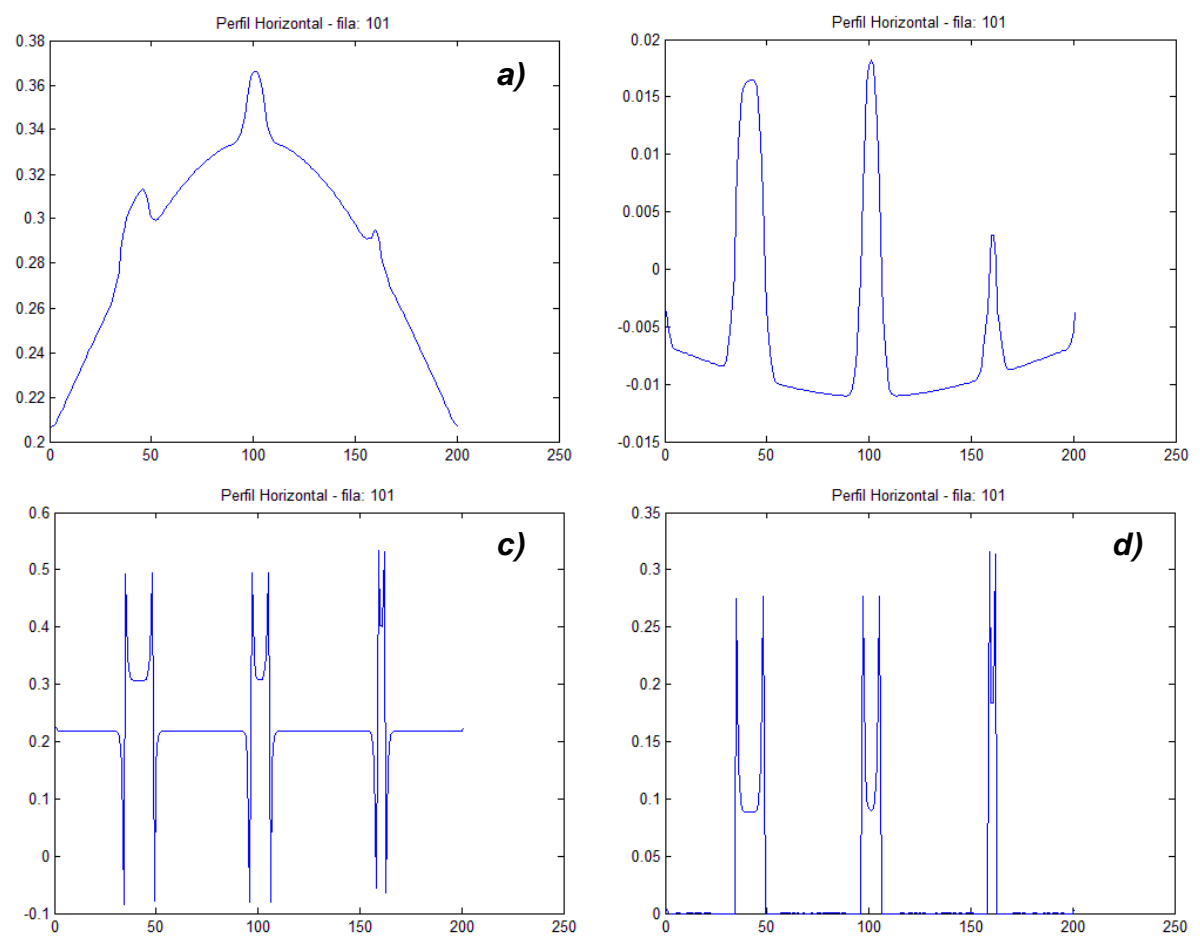

Fig. 8. Spatial profiles for thermogram at $t=5 s$, extracted from: a) original sequence; $b) D A C-m$ sequence; $c)$ FDTC; d) FDTC truncated.

The principal effect of those steeper edges generated after FDTC technique is a greater probability for detecting defects with a simple edge detection method, at least, under good SNR conditions. This effect is observed at figures 9a and $\mathrm{b}$, where the defect areas estimated by applying Laplacian detector on the maxigrams of the DAC-m and FDTC (with $1^{\text {st }}$ approx. and truncated) sequences, are shown.
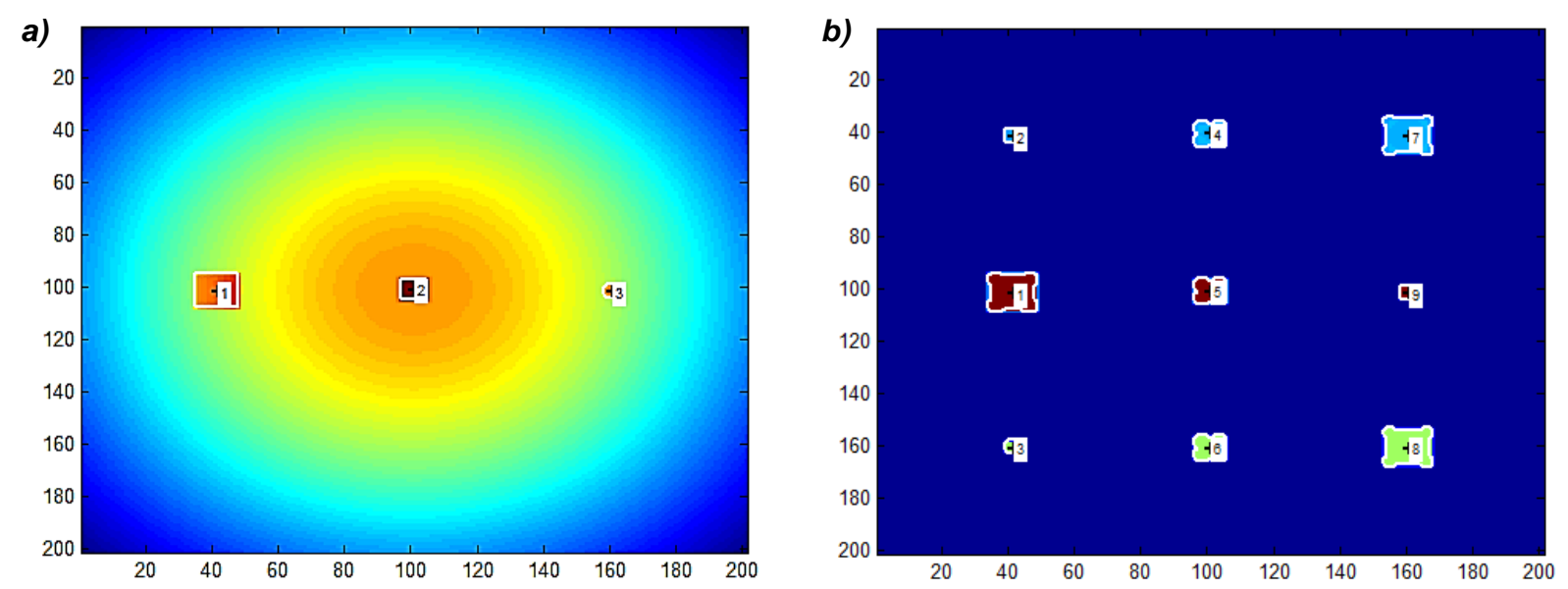

Fig. 9. Maxigrams and detected flaws from: a) DAC-m sequence; b) FDTC truncated.

The table 3 reveals the results of area estimation of detected flaws for each technique. This table proves that, under ideal conditions, FDTC method is able to detect the deeper and smaller defects, even with a relatively simple procedure, showing an acceptable performance in determining its areas, although the error increase with decreasing size/depth ratio. After detection of defect areas, the method to estimate their depths described in chapter 4, is tested with their centroids, obtaining a good agreement between simulated values and estimated ones, except for shallows defects; it is probably that the initial discrepancy between proposed model and synthetic data influences this estimation. In table 4 , results of depth estimation for different defects are listed. 
Table 3. Results of area estimation using Laplacian detector applied to the maxigram from DAC-m and FDTC sequences.

\begin{tabular}{|c|c|c|c|c|c|c|c|c|c|}
\hline & $\begin{array}{c}\text { Defect 1 } \\
{\left[\mathrm{mm}^{2}\right]}\end{array}$ & $\begin{array}{c}\text { Defect 2 } \\
{\left[\mathrm{mm}^{2}\right]}\end{array}$ & $\begin{array}{c}\text { Defect } 3 \\
{\left[\mathrm{~mm}^{2}\right]}\end{array}$ & $\begin{array}{c}\text { Defect } 4 \\
{\left[\mathrm{~mm}^{2}\right]}\end{array}$ & $\begin{array}{c}\text { Defect } 5 \\
{\left[\mathrm{~mm}^{2}\right]}\end{array}$ & $\begin{array}{c}\text { Defect } 6 \\
{\left[\mathrm{~mm}^{2}\right]}\end{array}$ & $\begin{array}{c}\text { Defect 7 } \\
{\left[\mathrm{mm}^{2}\right]}\end{array}$ & $\begin{array}{c}\text { Defect } 8 \\
{\left[\mathrm{~mm}^{2}\right]}\end{array}$ & $\begin{array}{c}\text { Defect } 9 \\
{\left[\mathrm{~mm}^{2}\right]}\end{array}$ \\
\hline Real area & 225 & 100 & 25 & 225 & 100 & 25 & 225 & 100 & 25 \\
\hline $\begin{array}{l}\text { Estimation } \\
\text { with DAC-m }\end{array}$ & 196 & 81 & 32 & 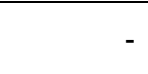 & - & - & - & & \\
\hline Error & $12.9 \%$ & $19 \%$ & $28 \%$ & - & - & - & - & - & - \\
\hline $\begin{array}{l}\text { Estimation } \\
\text { with FDTC }\end{array}$ & 228 & 113 & 36 & 220 & 113 & 32 & 220 & 113 & 36 \\
\hline Error & $1.3 \%$ & $13 \%$ & $44 \%$ & $2.2 \%$ & $13 \%$ & $28 \%$ & $2.2 \%$ & $13 \%$ & $44 \%$ \\
\hline
\end{tabular}

Table 4. Depth estimation of simulated flaws.

\begin{tabular}{|c|c|c|c|}
\hline Defect No. & Real depth [mm] & Estimated depth [mm] & Relative error [\%] \\
\hline $1,2,3$ & 0.20 & 0.26 & 30.0 \\
\hline $4,5,6$ & 0.60 & 0.58 & 3.3 \\
\hline $7,8,9$ & 1.00 & 0.93 & 7.0 \\
\hline
\end{tabular}

Finally, the table 5 shows runtimes for the DAC-m algorithm, the FDTC filtering (without considering truncation) and generation of complete temperature distribution throughout the slab by means of ThermoCalc6 $\mathrm{L}$ which is the base for former 3D filtering technique. For this test, a Core i7 PC with 8GB of RAM was used, running Windows7 and MATLAB.

Table 5. Runtimes for DAC-m, FDTC and $3 D$ filtering.

\begin{tabular}{|c|c|}
\hline Technique & Runtime [s] \\
\hline CAD-m & 0.9 \\
\hline FDTC & 2.1 \\
\hline 3D filtering & 83.0 \\
\hline
\end{tabular}

\section{Conclusions and future work}

In this paper has been introduced a new technique for contrast enhancement and characterization of defect areas from a sequence of thermal images captured through a PT experiment on a slab. The method proposed, named Finite Differences Thermal Contrast (FDTC) is based on a 3D discrete model of heat propagation to estimate the next temperature on each pixel according to the current temperature of the same pixel and its 4-neighbors, in such a way that these terms can be arranged in a spatial filtering mask. This filtering leads to compensate (under ideal conditions of acquisition) the thermal background non-uniformity to achieve optimum contrast of the defect regions, spend just twice the runtime for DAC-m, and around $2.5 \%$ the runtime for 3D complete reconstruction of temperature distribution throughout a slab like that used in tests.

After validation of the technique, using an artificial sequence of thermal images from a CFRP slab generated with the software ThermoCalc6L, it was found that in applying FDTC the thermal profiles of sound points rapidly remain quite flat (uniform background). Additionally, defects tend to retain their original shape despite the heat lateral spread which introduces a typical blur effect in the borders. Combining these two effects, it is possible to detect all simulated defects with a simple technique of edge detection. About defect area estimation, however, we have that the less size/depth ratio of a defect, the more relative error, although an improvement is obtained respect to DAC for shallow defects.

Finally, the results show quite appreciable improvement in the contrast of the defects by using the FDTC under absence of noise and optical distortions, meanly due to steeper edges and a slower decay of accumulated profiles amplitude with depth (figure 6). So, the principal future work will be the FDTC implementation to filtering real thermal sequences, where noise and distortions may reduce the performance of the technique. The use of accumulated profiles may help to reach a suitable SNR for defect detection; but anyway a good pre-filtering, or even a statistical adjust procedure, will be necessary to avoid bad results, overall, if the differential profiles in figure 4 are used for depth estimation of defects. 


\section{REFERENCES}

[1] Snell Jr, J., Spring, R. "Infrared Thermography Advances". Featured Articles - NDT Database \& Journal, October 2007. Consulted on November 2010 from: http://www.ndtmag.com/Articles/Feature Article/BNP GUID 9-5-2006 A 10000000000000179212

[2] Pohl, J. "Ultrasonic Inspection of Adaptive CFRP-Structures". NDT Database \& Journal, Vol.3, No.9, September 1998. Consulted on February 2012 from: http://www.ndt.net/article/ecndt98/aero/015/015.htm

[3] IATA - International Air Transport Association. "A global approach to reducing aviation emissions. First stop: carbon-neutral growth from 2020". Switzerland, 2009.

[4] Ibarra-Castanedo, Clemente. "Quantitative Subsurface Defect Evaluation by Pulsed Phase Thermography: Depth Retrieval with the Phase". PhD Thesis. Université Laval, Faculté des Sciencies et de Génie. Quebec, 2005.

[5] Grinzato, E., Bison, P.G., Marinettti, S., Vavilov, V. "Thermal NDE enhanced by 3D numerical modeling applied to works of art”. $15^{\text {th }}$ World Conference on Non-destructive Testing, Rome, Italy 2000. Consulted on December 2008, from: http://www.ndt.net/article/wcndt00/papers/idn909/idn909.htm

[6] Benítez, H., Ibarra, C., Bendada, A., Maldague, X., Loaiza, H., Caicedo, E. "Modified Differential Absolute Contrast Using Thermal Quadrupoles for the Nondestructive Testing of Finite Thickness Specimens by Infrared Thermography". Canadian Conference on Electrical and Computer Engineering. Ottawa, Canada, 2006.

[7] Valdes, M., Inamura, M., Valera, J., Yao, L. "Multidimensional filtering approaches for pre-processing thermal images". Multidimensional System Signal Processing, p.299-325. Springer Science+Business Media, 2006.

[8] Alsaadawi, Z., Netzelmann, U. "Spatio-temporal filtering of active thermography data for noise reduction and data compression". 10 International Conference on Quantitative Infrared Thermography, July, 2010, Quebec, Canada.

[9] Cheng-Hung $\mathrm{H}$., Meng-Ting $\mathrm{C}$. "A transient three-dimensional inverse geometry problem in estimating the space and time-dependent irregular boundary shapes". International Journal of Heat and Mass Transfer, Vol. 51, p. 5238-5246. Elsevier, 2008.

[10] Benítez, H., Loaiza, H., Caicedo, E. "Termografía Activa Pulsada en Inspección de Materiales. Técnicas Avanzadas de Procesado". Colección de Ciencias Físicas, Exactas y Naturales. $1^{a}$ edición. Cali, Colombia. Marzo de 2011.

[11] Maldague, Xavier (editor técnico). "Infrared and Thermal Testing". Nondestructive Testing Handbook, Vol. 3, $3^{\text {rd }}$ edition, American Society for Non Destructive Testing, p.719. USA, 2001. CD-ROM. ISBN 1-57117-081-5.

[12] Darabi, Akbar. "Detection and Estimation of Defect Depth in Infrared Thermography Using Artificial Neural Networks and Fuzzy Logic". 167 p. PhD Thesis. Université Laval, Faculté des Sciencies et de Génie. Quebec, 2000.

[13] Hensen, J.L.M. \& Nakhi, A. (1994). Fourier and Biot numbers and the accuracy of conduction modeling. Proceedings of BEP'94 Conference "Facing the Future", April, 1994. pp. 247-256. York: Building Environmental Performance Analysis Club (BEPAC)

[14] Tadeu, A., Simões, N. "Fundamental solutions for transient heat transfer by conduction and convection in an unbounded, half-space, slab and layered media in the frequency domain". Engineering Analysis with Boundary Elements, Vol. 29, pp.1130-1142. Elsevier, 2005.

[15] Tadeu, A., Simões, N. "Three-dimensional fundamental solutions for transient heat transfer by conduction in an unbounded medium, half-space, slab and layered media". Engineering Analysis with Boundary Elements, Vol. 30, pp.338-349. Elsevier, 2006.

[16] Tadeu, A., Simões, N. "Conduction and convection phenomena through a slab with thermal heterogeneities". Applied Mathematical Modeling, Vol. 31, 1444-1459. Elsevier, 2007.

[17] Gilbert, S., Mathias, R. "Analysis of diffusion delay in a layered medium. Application to heat measurements from muscle”. Biophysical Journal, Vol. 54, p. 603-610. October 1988. 